\title{
Oculopharyngeal Muscular Dystrophy Associated with Dementia
}

\author{
Yoshikazu Mizoi ${ }^{1}$, Toshimasa Yamamoto ${ }^{1}$, Narihiro Minami ${ }^{2}$, Aya Ohkuma ${ }^{1}$, Ikuya Nonaka ${ }^{2}$, \\ Ichizo Nishino ${ }^{2}$, Naotoshi Tamura ${ }^{1}$, Takahiro Amano ${ }^{3}$ and Nobuo Araki ${ }^{1}$
}

\begin{abstract}
We report genetically confirmed heterozygote oculopharyngeal muscular dystrophy (OPMD) accompanied by dementia, suggesting a possible causal association between OPMD and dementia. The proband first noticed bilateral ptosis, dysphagia, and proximal dominant muscle weakness in the lower extremities at age 53. Ten years later, she was found to have dementia with a score of 10/30 on the mini-mental state examination (MMSE). On PABPN1 gene analysis, the GCN repeat was expanded 17 times in one allele. In addition, the proband's younger brother exhibited myopathy and dementia. To our knowledge, this is the first report of genetically confirmed heterozygote OPMD associated with dementia.
\end{abstract}

Key words: oculopharyngeal muscular dystrophy (OPMD), heterozygote, expanded PABPNI (GCN) 17 mutation, dementia

(Intern Med 50: 2409-2412, 2011)

(DOI: 10.2169/internalmedicine.50.5577)

\section{Introduction}

Oculopharyngeal muscular dystrophy (OPMD) (1) is an autosomal dominant muscle disease caused by an increase in GCN repeats in the polyadenylate binding protein nuclear 1 (PABPN1; also called PABP2) gene (2), which is located on 14q11.2-q13 (3). Normally there are $10 \mathrm{GCN}$ repeats, but they are increased 12-17 times in OPMD (4).

The disease usually starts in the fifth decade of life or later, and is clinically characterized by ptosis, mild ophthalmoplegia, dysphagia, and generalized muscle weakness and atrophy. Little attention has been paid to central nervous system (CNS) involvement in OPMD. To our knowledge, two reports have described OPMD patients with mental retardation $(5,6)$, one with cognitive impairment $(7)$, one with spinal cord involvement (8).

Here, we report genetically confirmed heterozygote OPMD accompanied by dementia.

\section{Case Report}

\section{Patient 1 (proband)}

A 63-year-old woman without an informative medical history and with no vascular risk factor profiles (e.g., diabetes mellitus, hypertension, smoking) was admitted to the hospital due to symptoms of progressive ptosis, dysarthria, and limb muscle weakness in March 2007. She was the product of nonconsanguineous parents and her perinatal history and developmental course were normal. She finished high school, worked in an office, and was married. She developed bilateral ptosis, dysarthria, and gait disturbance 10 years before admission. At one point, she had received a diagnosis of progressive supranuclear palsy or myasthenia gravis at another hospital; however, she refused further investigation. At age 61, she developed dysphagia without aspiration. According to her husband, her intelligence seemed normal at that time. She had also been suffering from arteriosclerosis obliterans (ASO) with an infection and skin ulcer in the lower extremities for 6 weeks before admission.

\footnotetext{
${ }^{1}$ Department of Neurology, Faculty of Medicine, Saitama Medical University, Japan, ${ }^{2}$ National Institute of Neuroscience, National Center of Neurology and Psychiatry, Japan and ${ }^{3}$ Department of Medical Educational Center, Faculty of Medicine, Keio University, Japan Received for publication March 30, 2011; Accepted for publication July 10, 2011

Correspondence to Dr. Yoshikazu Mizoi, mizoi_y@saitama-med.ac.jp
} 


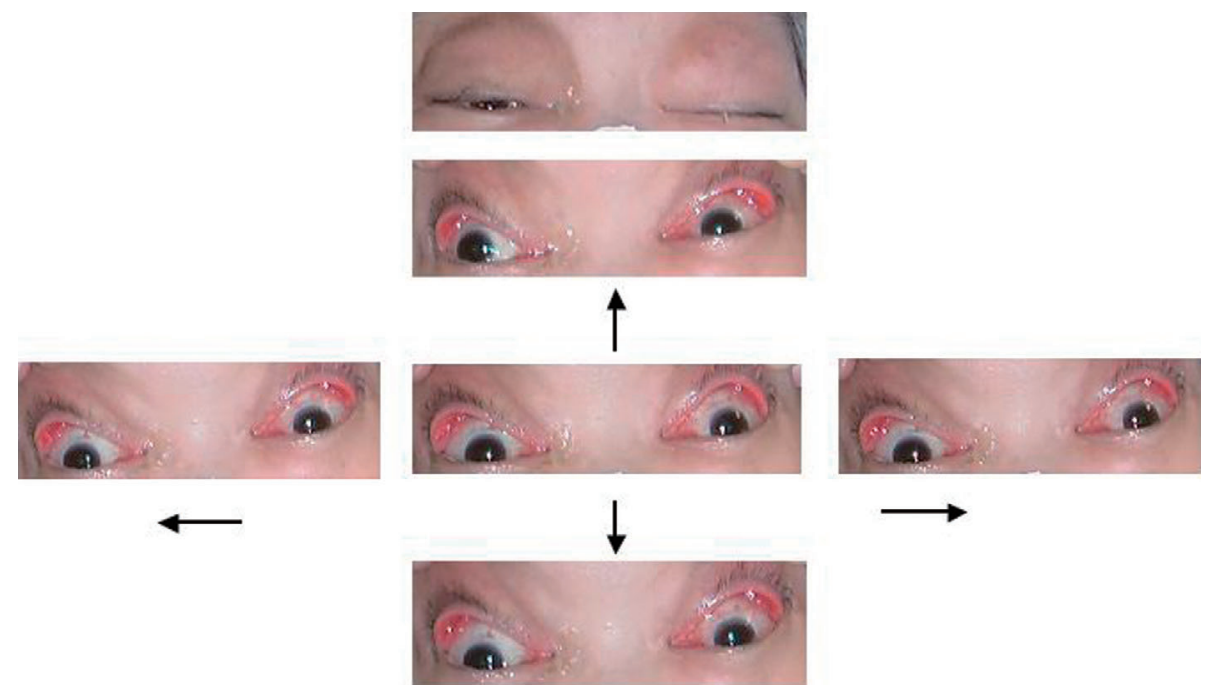

Figure 1. Proband uses her fingers to lift her eyelids due to marked bilateral ptosis and external ophthalmoplegia.

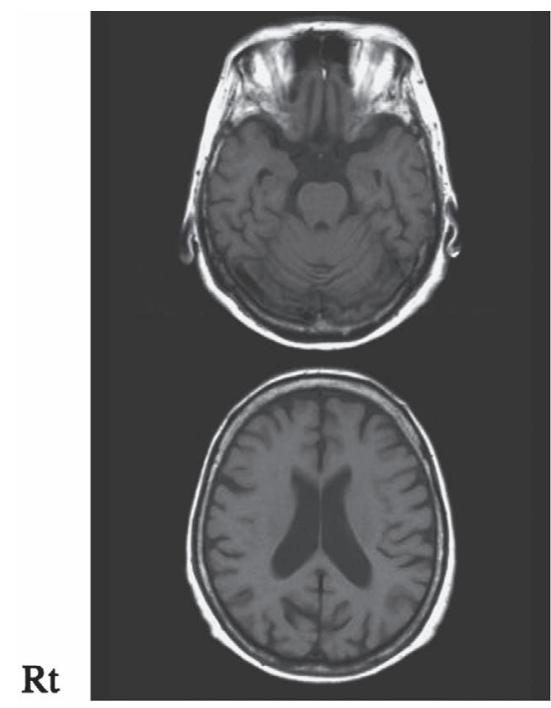

T1WI

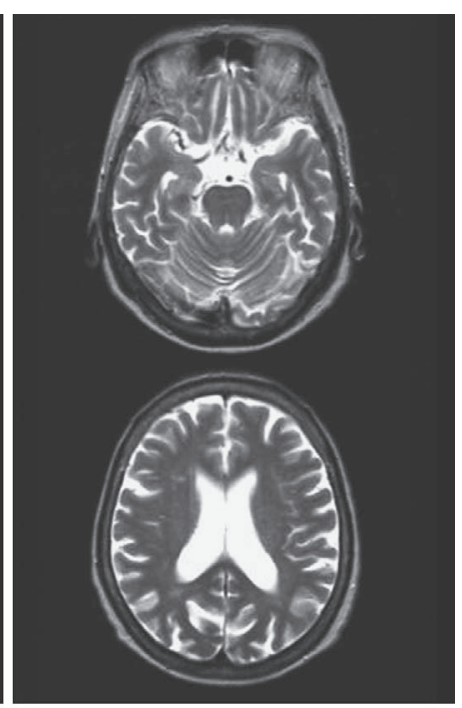

T2WI

Figure 2. MRI of proband shows neither abnormalities nor parietal lobe atrophy. Hippocampal volume is normal on VSRAD.

With regard to family history, the father of Patient 1 had generalized muscle weakness and was wheelchair-bound in his fifth decade; he died in his seventh decade. Her three siblings (two brothers, ages 66 and 61, and a sister, age 65), were reported to have muscle weakness, suggesting autosomal-dominant inheritance. Her younger brother (patient 2) was admitted to another hospital at age 45 due to muscle weakness. (Information on patient 2 is provided below.) Her husband did not supply further information about her educational background or cognitive impairment of her family members.

On admission, Patient 1 had gangrene on the bilateral distal lower extremities with ASO. She was conscious and alert. She had to lift her eyelids with her fingers due to marked bilateral ptosis and external ophthalmoplegia (Fig. 1). She also had facial muscle weakness, a nasal voice, dysarthria, and dysphagia, which necessitated the insertion of a nasogastric tube. Diffuse limb muscle weakness and atrophy were noted and respiratory function was normal. Her intellectual level was markedly decreased with severe disorientation in regard to time and place. She also had poor word recall, comprehension, and calculation; her mini-mental state examination (MMSE) was 10/30. Her level of cooperation did not allow further cognitive evaluation.

Blood chemistry was uninformative; values of electrolytes, CK, AST, ALT, thyroid hormones, thiamine, lactic acid, pyruvic acid, acetylcholine receptor antibody, and Jo-1 antibody were all within normal ranges. Arterial blood gas and cerebrospinal fluid were normal. Total tau-protein and amyloid beta protein in the cerebrospinal fluid $(9,10)$ were also within normal ranges.

On electromyography, the facial muscles, upper extremi- 


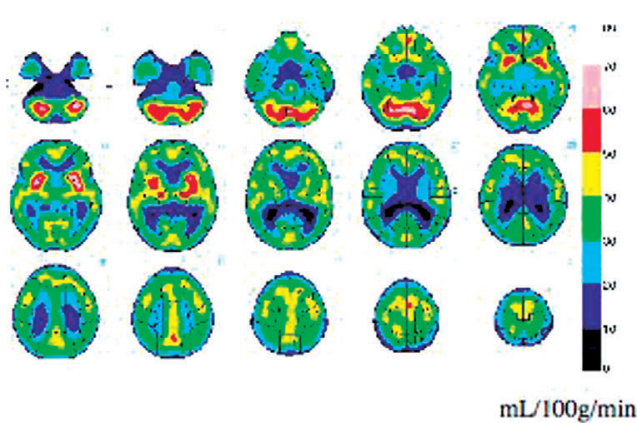

A

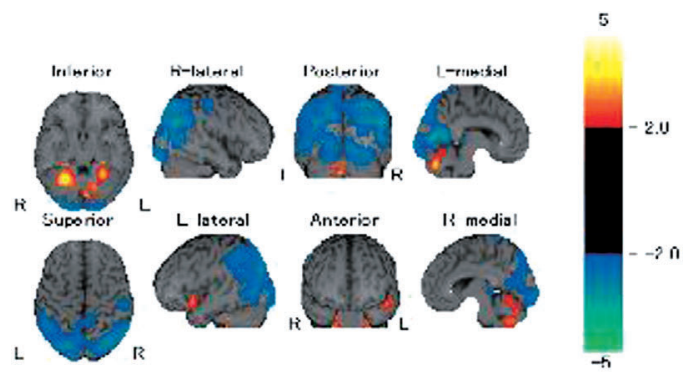

Z-score

B

Figure 3. Proband's SPECT and eZIS. eZIS reveals a decrease in cerebral blood flow at the bilateral parietal and occipital lobe (B), and SPECT does a relative increase at the bilateral basal ganglia, thalamus, and cerebellum (A).

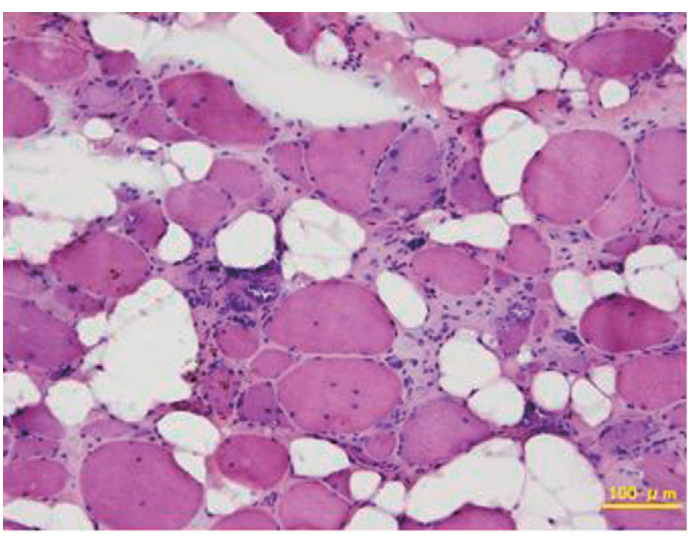

A

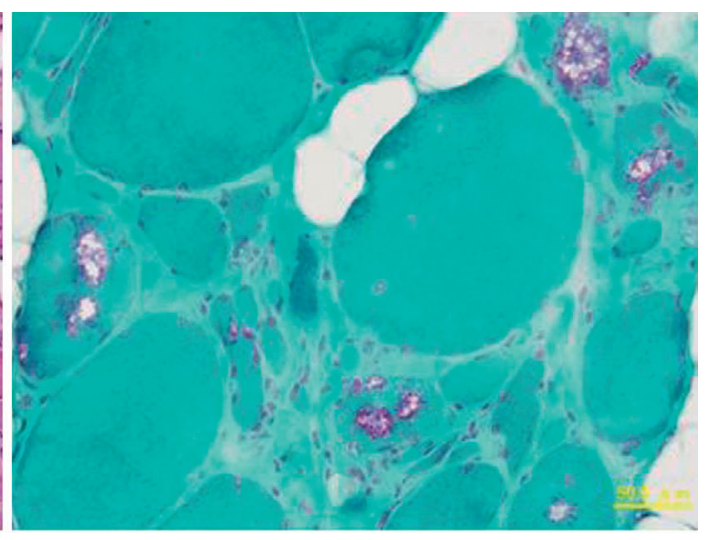

B

Figure 4. Left pectoralis major muscle pathological findings. Fiber size variation (A: Hematoxylin and Eosin staining) and scattered rimmed vacuoles (B: modified Gomori trichrome staining) are visible.

ties, and diaphragm showed short-duration, polyphasic motor unit potentials with early recruitment. Motor and sensory conduction studies revealed normal results. No epileptiform waves were identified on electroencephalogram. Orbital CT and brain MRI showed no abnormalities (Fig. 2). Hippocampal volume was normal on voxel-based specific regional analysis for Alzheimer's disease (VSRAD) (11). ${ }^{123} \mathrm{I}-$ meta-iodobenzylguanidine (MIBG) myocardial scintigraphy $(12,13)$ did not reveal cardiac sympathetic denervation. Single photon emission tomography (SPECT) with easy Zscore imaging system (eZIS) (14) revealed a decrease in cerebral blood flow at the bilateral parietal and occipital lobe and a relative increase at the bilateral basal ganglia, thalamus, and cerebellum (Fig. 3).

Doppler ultrasonography, enhanced CT, and MRI of the body showed occlusion in the distal abdominal aorta and bilateral common iliac arteries, and arterial flow on the distal portion of her bilateral legs was extremely decreased, indicating Leriche syndrome due to ASO. Doppler ultrasonography of the upper extremities was relatively normal. Concerning the carotid arteries, an intimal-medial thickness at the right common carotid artery was $3.3 \mathrm{~mm}$, and that at the left was $1.7 \mathrm{~mm}$, suggesting mild arteriosclerosis. Axillobifemoral bypass was immediately performed, and with written informed consent, a muscle biopsy was taken from the left pectoralis major muscle.

Muscle pathology revealed scattered rimmed vacuoles in addition to fiber size variation (Fig. 4). There were no visible ragged red fibers, cytochrome $c$ oxidase deficient fibers, or strongly succinate dehydrogenase-reactive blood vessels. The analysis of the GCN repeats region in PABPN1 gene by capillary electrophoresis of PCR products revealed 17/10 $\mathrm{GCN}$ repeats $\left[(\mathrm{GCG})_{6} \mathrm{GCA}(\mathrm{GCG})_{6}(\mathrm{GCA})_{3} \mathrm{GCG} /(\mathrm{GCG})_{6}\right.$ $(\mathrm{GCA})_{3}(\mathrm{GCG})$ ], leading to a diagnosis of OPMD. The two daughters of Patient 1, aged 35 and 32, had normal neurological physical examinations. The other family members were uncooperative and did not agree to gene tests.

\section{Patient 2 (younger brother of Patient 1)}

Patient 2 developed muscle weakness of the extremities with distal dominance at age 43, and was admitted to another hospital 2 years later, in January 1992. After finishing high school, he worked as a taxi driver. Patient 2 did not give a detailed family history. On admission, he was con- 
scious and alert. He did not show any obvious blepharoptosis, external ophthalmoplegia, dysarthria, or difficulty swallowing, but diffuse, mild muscle weakness and atrophy were present. He walked with a waddling gait, and Gowers' sign was positive. Blood chemistry and cerebrospinal fluid were uninformative. Muscle biopsy from the left biceps brachii muscles revealed scattered fibers with rimmed vacuoles in addition to variation in fiber size, which led to a diagnosis of distal myopathy with rimmed vacuoles (DMRV) at that time. Gene analysis for OPMD had not yet been established. He also had dementia with a total IQ of 72 (verbal 80; performance 68) on the Wechsler Adult Intelligence ScaleRevised. Brain MRI showed no particular abnormalities, but SPECT revealed a diffuse decrease in cerebral blood flow before eZIS was established.

\section{Discussion}

To our knowledge, this is the first report of genetically confirmed heterozygote OPMD with CNS involvement. Patient 2 did not show a typical clinical picture with ophthalmoplegia or dysphagia, but he likely had OPMD, as his muscle pathology was identical to that of OPMD.

The dementia in Patient 1 was likely caused by a unique pathomechanism. Alzheimer's disease, dementia with Levy bodies, vascular dementia, and metabolic dementia were clinically unlikely and all laboratory indicators for these dementias were negative. The fact that her brother also had similar clinical and pathological manifestations, though genetically unconfirmed, raises the possibility that OPMD and a unique form of dementia are causally related. A few previous reports have mentioned possible mental retardation $(5,6)$ and spinal cord involvement $(8)$ in OPMD, although a diagnosis was not genetically confirmed in these cases. Recently, cognitive impairment was reported in association with homozygote OPMD (7). The authors reviewed the charts of 10 patients previously diagnosed as homozygotes for the (GCG)13 mutation producing OPMD. One patient among those reported had undergone a neuropsychological evaluation, which revealed mild to moderate cognitive impairment, but none of the heterozygote parents of any of the 10 patients exhibited mental or mood disturbances. In the case of Patient 1 (proband), the brain MRI did not show parietal cortical atrophy but eZIS revealed a decrease in cerebral blood flow at the parietal lobe, which suggested involvement. We understand this discrepancy between the brain image and function to be a reflection of hypometabolism in those regions. The present case was heterozygote OPMD with an expanded PABPN1 (GCN) $17 \mathrm{mu}-$ tation.

In support of this hypothesis, transgenic mice expressing an expanded GCG repeat in the Pabpnl gene showed ubiquitinated Pabpnl positive intranuclear inclusions in brain neurons and Purkinje cells (15). It is possible that similar pathological changes occur in the human brain. Autopsy cases of brain pathology may help to clarify this question.

The authors state that they have no Conflict of Interest (COI).

\section{References}

1. Brais B, Tome E. Oculopharyngeal muscular dystrophy. In: Myology. Engel A, Laurea C, Eds. McGraw-Hill, New York, 2004: 1147-1162.

2. Brais B, Bouchard JP, Xie YG, et al. Short GCG expansion in the PABPN2 gene case oculopharyngeal muscular dystrophy. Nat Genet 18: 164-167, 1998.

3. Brais B, Xie YG, Sanson M, et al. The oculopharyngeal muscular dystrophy locus maps to the region of the cardiac alpha and beta myosin heavy chain gene on chromosome 14q 11.2-q13. Hum Mol Genet 4: 429-434, 1995.

4. Brais B, Rouleau G. Oculopharyngeal Muscular Dystrophy. Gene Reviews, Pagon RA, Bird TD, Dolan CR, Stephens K, Eds. 2006 http://www.genetests.org

5. Millefiorini M, Filippini C. Oculopharyngeal muscular dystrophy. Riv Neurol 37: 327-337, 1967 (in Italian, Abstract in English).

6. Sarkar AK, Biswas SK, Ghosh AK, et al. Oculopharyngeal muscular dystrophy. Indian J Pediatr 62: 496-498, 1995.

7. Blumen SC, Bouchard JP, Brais B, et al. Cognitive impairment and reduced life span of oculopharyngeal dystrophy homozygotes. Neurology 73: 596-601, 2009.

8. Linoli G, Tomelleri G, Ghezzi M. Oculopharyngeal muscular dystrophy. Description of a case with involvement of the central nervous system. Pathologica 83: 325-334, 1991 (in Italian, Abstract in English).

9. Arai H, Terajima M, Miura M, et al. Tau in cerebrospinal fluid. A potential diagnostic marker in Alzheimer's disease. Ann Neurol 38: 649-652, 1995

10. Mottter R, Vigo-Pefrey C, Kholodenko D, et al. Reduction of beta-amyloid peptide 42 in the cerebrospinal fluid of patients with Alzheimer's disease. Ann Neurol 38: 643-648, 1995.

11. Hirata $Y$, Matsuda $H$, Nemoto K, et al. Voxel-based morphometry to discrminate early Alzheimer's disease from controls. Neurosci Lett 382: 269-274, 2005.

12. Watanabe $H$, Ieda $T$, Katayama $T$, et al. Cardiac ${ }^{123} I$-metaiodobenzyl guanidine (MIBG) uptake in dementia with Lewy bodies. Comparison with Alzheimer's disease. J Neurol Neurosurg Psychiatry 70: 781-783, 2001.

13. Yoshida M, Taki J, Yamada M. A clinical role for ${ }^{123}$ I MIBG myocardial scintigraphy in the distinction between dementia of Alzheimer's-type and dementia with Lewy bodies. J Neurol Neurosurg Psychiatry 71: 583-588, 2001.

14. Matsuda H, Mizumura S, Nagao T, et al. Automated discripmination between very early Alzheimer disease and control using an easy Z-score imaging system for multicenter brain perfusion single-photon emission tomography. Am J Neuroradiol 28: 731736, 2007.

15. Dion P, Shanmugam V, Gaspar C, et al. Transgenic expression of an expanded (GCG)13 repeat PABPN1 leads to weakness and coordination defects in mice. Neurobiological Disease 18: 528-536, 2005.

(C) 2011 The Japanese Society of Internal Medicine http://www.naika.or.jp/imindex.html 